\title{
Radiocarbon
}

1973

\section{ANU RADIOCARBON DATE LIST V}

\author{
MICHAEL BARBETTI and HENRY POLACH \\ Dept. of Gcophysics and Geochemistry \\ Australian National University, Canberra, Australia
}

The present date list contains only details of $\mathrm{C}^{14}$ measurements on sites selected for archaeomagnetic study. Except where otherwise stated in the text, all samples were collected in 1970 or 1971 during fieldwork by one of the authors (M.B.), and were pretreated with hot $2 \mathrm{~N} \mathrm{HCl}$ to remove any possible contamination by pedogenic carbonate. Benzene samples were prepared using updated synthesis techniques (Polach et al., 1972) and measurements of $\mathrm{C}^{14}$ activity were made on two Beckman LS-200 liquid scintillation spectrometers following automatic cycling procedures described previously (Polach, 1969). Samples ANU-677-697 were counted on the spectrometer which has been in use since 1968 (LS-1).

The other samples (ANU-651-676) were counted on the second spectrometer (LS-2). We made a series of measurements of background activity with new counting vials before and after measuring the activities of the samples and the activity of a tree-ring sample (Cryptomeria Japonica, growth intervals A.D. 1890-1900) provided by K. Kigoshi, Gakushuin Univ., Tokyo, Japan. The measured tree-ring activity, corrected for isotopic fractionation (using $\delta \mathrm{C}^{1.3}=-23.6 \pm 0.5$, suggested by K. Kigoshi) and radioactive decay (between A.p. 1895 and 1950), has been used as the modern standard activity for LS-2. The activity of that wood correlated well with the NBS oxalic standard activity on LS-I. (Polach, 1972). Table 1 compares the activities of duplicate samples counted on LS-1 and LS-2.

The values agree well within their errors, and we concluded that agreement between LS-1 and LS-2 was satisfactory.

Table 2 lists the measured $\delta \mathrm{C}^{13}$ values of four samples from the Willandra Lakes scries. The values suggest to us that a $\delta \mathrm{C}^{13}$ value of $-22 \pm 2 \%$ is appropriate for charcoal from the semi-arid zone of Australia, and we have used this value for the remaining samples of the Willandra Lakes series and the Partacoona scries. Elsewhere we have used a $\delta \mathrm{C}^{13}$ value of $-24 \pm 2 \%$. Such estimated values are quoted as est. $-22 \%$ or est. $-24 \%$ in the text.

\section{ACKNOWLEDGMENTS}

We thank H. Allen, J. M. Bowler, D. Edwards, F. E. M. Lilley, M. W. McElhinny, D. J. Mulvaney, and J. Urquhart, Australian National 
University; A. Gallus and members of the Archaeological Society of Victoria, T. Langford-Smith, University of Sydney, A. Barnes of Mungo Station, and B. Powell of Partacoona Station for their assistance with fieldwork; J. Head and J. Gower for their help in the laboratory; and J. M. Bowler for providing the base map for Fig. 1 .

TABLE 1

Activities of duplicate benzene samples measured by different liquid scintillation spectrometers.

(Results generally constitute good statistical agreement)

\begin{tabular}{lllc}
\hline $\begin{array}{l}\text { Sample } \\
\text { no. }\end{array}$ & $\begin{array}{c}\text { LS-1 } \\
\text { measured } \delta \mathrm{C}^{14}\end{array}$ & $\begin{array}{c}\text { LS-2 } \\
\text { measured } \delta \mathrm{C}^{1-4}\end{array}$ & $\begin{array}{c}\text { Accepted mean } \delta \mathrm{C}^{14} \\
\text { reported in this } \\
\text { date list }\end{array}$ \\
\hline ANU-087 & $-187.4 \pm 6.7$ & $-179.1 \pm 5.2$ & $-181.4 \pm 3.8$ \\
& $-176.3 \pm 10.1$ & & $-101.8 \pm 4.1$ \\
ANU-387 & $-114.4 \pm 6.9$ & $-97.5 \pm 5.5$ & -14.5 \\
ANU-447 & $-74.1 \pm 14.5$ & $-996.1 \pm 2.3$ & $\begin{array}{c}\text { values differ } \\
\text { significantly* }\end{array}$ \\
ANU-448 & $-976.2 \pm 3.0$ & & $-989.0 \pm 1.7$ \\
ANU-656 & $-19.1 \pm 4.2 \pm 2.0$ & $-986.0 \pm 3.4$ & $-14.5 \pm 3.3$ \\
ANU-660 & $-107.7 \pm 3.9$ & $-100.7 \pm 7.4$ & $-106.1 \pm 3.4$ \\
ANU-672 & $-27.1 \pm 4.0$ & $-16.8 \pm 5.6$ & $-23.7 \pm 3.3$
\end{tabular}

* Discrepancy may have been due te slightly different pretreatment of samples.

TABLE 2

Values of $\delta \mathrm{C}^{13}$

\begin{tabular}{cc}
\hline Sample & $\delta \mathrm{C}^{13} \pm 0.2 \% 0^{*}$ \\
\hline ANU-651 & -22.8 \\
ANU-660 & -22.5 \\
ANU-674 & -20.9 \\
ANU-680 & -22.5 \\
\hline
\end{tabular}

* w.r.t. P. D. Belemnite carried out by Krucger Lnterprises, Cambridge, Mass.

\section{SAMPLE DESCRIPTIONS}

\section{Willandra Lakes series}

$A$ chain of ancient lakes in W New South Wales forms the terminal system of Willandra Creek, a distributary stream which leaves the Lachlan R. some $250 \mathrm{~km}$ to the E. The Willandra Creek now carries water only when major flooding of the Lachlan occurs, but in Pleistocene time its flow was sufficient to maintain a high water level in the lakes for long periods. The lakes themselves have been dry for the last 15,000 yr (Bowler, 1971). 
The Quaternary geology of the lakes has been described by Bowler ( 1971 ); we present a brief summary. In plan, the lakes have cliffed $W$ margins and long transverse dunes (lunettes) around their $\mathrm{E}$ shores. Three soil-stratigraphic units are recognized in the lunettes. The basal Golgol unit consists of a deep, red calcareous soil developed on quartz sands, and is beyond the range of radiocarbon dating. The lakes were active by at least 40,000 B.P., and subsequent high water levels resulted in deposition of the quartz sands of the Mungo unit. By ca. 25,000 yr B.P. the lakes were almost dry, and in many places calcareous and argillaceous silty sands were deposited in the lunette. The top of the thin Mungo unit is marked by soil development, overlain by a deposit (up to $32 \mathrm{~m}$ thick) of aeolian silt with well-preserved bedding (Zanci unit), deposited rapidly during the final drying of the lakes between 17,500 and 16,000 yr B.P.

Fig. 1 shows a plan of the last 4 Willandra Lakes, and the locations of the archacomagnetic sites for which dates are presented here.

Preliminary archaeomagnetic measurements on baked earth and cooking stones from 5 Aboriginal fireplaces at Lake Mungo (ANU-677, -680-683) show that there was an unusually large geomagnetic excursion 30,000 yr B.1', with the field rotating $>120^{\circ}$ away from its present attitude (Babetti and McElhinny, 1972). The relationship of these fireplaces and the luncte stratigraphy was previously described (Barbetti and Allen, 1972).

\section{ANU-651.}

\section{A. Lake Arumpo}

$$
\begin{aligned}
\delta C^{14} & =-8.8 \pm 7.9 \\
\Delta & =+4.3 \pm 7.9
\end{aligned}
$$

$>$ Modern

$\delta C^{13}=-22.8 \pm 0.2 \%$

Cilarcoal from Aboriginal oven on low rise, floor of (middle) Lake Cilibnalwool. Benzene dilution, $2060 \mathrm{~min}$. count. Indicated contemporary age mity be result of fine rootlet contamination which was not necessarily all removed during pretreatment.

ANU-670.

$$
\begin{array}{rrr}
\delta C^{14}=-963.2 \pm 8.6 & \mathbf{2 6 , 5 8 0}{ }^{+2130} \\
\Delta & =-963.1 \pm 8.5 \quad \text { Est. } \delta C^{1.3}=-22 \% 0
\end{array}
$$

Charcoal concentration in topmost clay sediment of Outer Arumpo lunette. Benzene dilution, $2760 \mathrm{~min}$. count.

ANU-671.

$$
\begin{array}{rlrl}
\delta C^{14} & =-154.2 \pm 7.4 & \mathbf{1 3 9 0} \pm \mathbf{8 0} \\
\Delta & =-159.3 \pm 8.1 & \text { Est. } \delta C^{1.3}=-22^{\prime} / \mathrm{cos}
\end{array}
$$
Charcoal from Aboriginal oven covered by loose sand on top of Outer Arumpo lunette. Benzene dilution, $1020 \mathrm{~min}$. count.

ANU-672.

$$
\begin{array}{rlr}
\delta C^{14} & =-23.7 \pm 3.3 & \mathbf{2 4 0} \pm \mathbf{6 0} \\
\Delta & =-29.5 \pm 5.1 & \text { Est. } \delta C^{13}=-22 \%
\end{array}
$$

Charcoal from Aboriginal oven in sand on top of Outer Arumpo lunette. Result is error weighted mean of 2 independent determinations: 
ANU-672/1 $\left(\delta \mathrm{C}^{14}=-27.1 \pm 4.0,270 \pm 50\right.$ B.P. $)$ and ANU-672/2 $\left(\delta \mathrm{C}^{14}\right.$ $=-16.8 \pm 5.6,180 \pm 60$ B.P.). Benzene $6080 \mathrm{~min}$. total count.

ANU-688.

$$
\begin{aligned}
& \delta C^{14}=-986.8 \pm 1.7 \\
& \Delta=-986.9 \pm 1.7
\end{aligned}
$$

Charcoal from area of burnt sand overlain by $3 \mathrm{~m}$ clay sediment, exposed in a gully in Outer Arumpo lunette. Benzene dilution, 4220

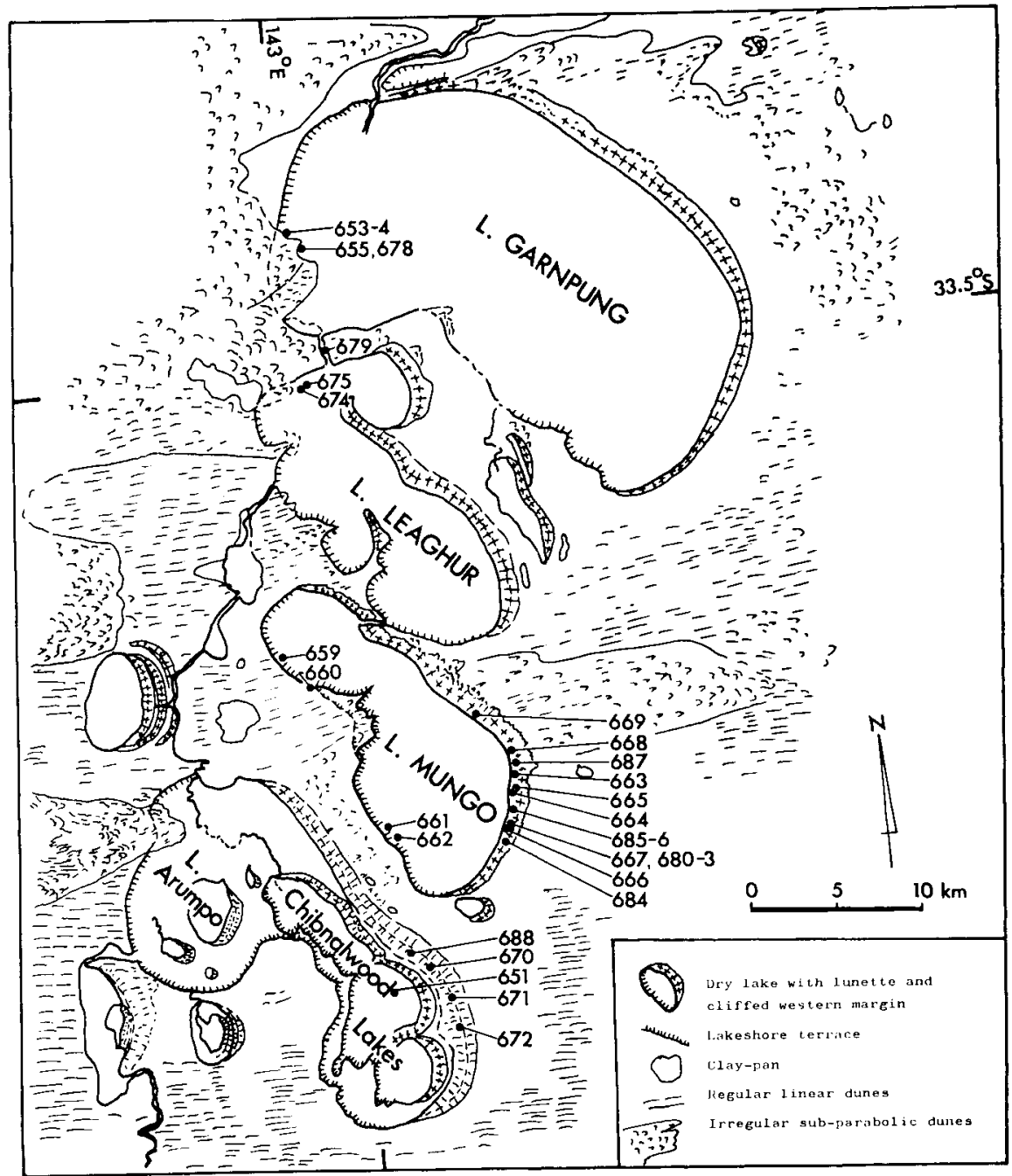

Fig. 1. Site locations for Willandra Lakes series, identificd by radiocarbon (ANU) sample numbers. (Base map with geomorphic detail provided by J. M. Bowler). 
min count. Charcoal pieces dated were encountered during excavation of archaeomagnetic samples for site previously dated (R., v. 12, p. 13:

ANU-304, $\Delta=-965.0 \pm 11.2,26,900+23100$ B.P.). Ages are in statistical agreement; better precision obtained with ANU-688.

\section{B. Lake Mungo}

ANU-659.

$$
\begin{array}{rrr}
\delta C^{14}=-84.4 \pm 16.9 & \mathbf{7 6 0} \pm \mathbf{1 5 0} \\
\Delta=-89.8 \pm 17.2 & \text { Est. } \delta C^{13}=-22 \% 0
\end{array}
$$

Charcoal from Aboriginal oven on sloping $W$ shore, $6 \mathrm{~m}$ above lake floor. Microlithic stone implements found nearby (H. Allen, pers. commun.). Benzene dilution, $1020 \mathrm{~min}$. count.
ANU-660.
$\delta C^{14}=-106.1 \pm 3.4$
$\Delta=-110.6 \pm 3.4$
$940 \pm 50$
$\delta C^{13}=-22.5 \pm 0.2 \%$

Charcoal from Aboriginal oven on plain overlooking $W$ margin of lake. Clay ovenstones were coated with secondary carbonate crust. Microlithic stone artifacts found nearby (H. Allen, pers, commun.). Result is error weighted mean of 2 independent determinations: ANU-660/1 $\left(\delta \mathrm{C}^{1.1}=-107.7 \pm 3.9,960 \pm 40\right.$ B.P. $)$ and ANU $660 / 2\left(\delta \mathrm{C}^{14}=-100.7 \pm\right.$ $7.4,890 \pm 70$ в.P.) Benzene dilution, $4380 \mathrm{~min}$. total count.

ANU-661.

$$
\begin{array}{rlr}
\delta C^{14} & =-176.9 \pm 10.5 & \mathbf{1 6 1 0} \pm \mathbf{1 1 0} \\
\Delta & =-181.9 \pm 11.0 & \text { Est. } \delta C^{13}=-22 \%
\end{array}
$$

Charcoal from Aboriginal oven on lake floor. $300 \mathrm{~m}$ from cliffed margin of lake. Benzene dilution, 1100 min. count.

ANU-662.

$$
\begin{aligned}
\delta C^{14} & =-232.7 \pm 6.9 \\
\Delta & =-237.3 \pm 7.6
\end{aligned}
$$$$
2180 \pm 80
$$$$
\text { Est. } \delta C^{13}=-22 \%
$$

Charcoal from small area of burnt clayey earth, preserved underneath $50 \mathrm{~cm}$ stabilized sand. Lake floor, $300 \mathrm{~m}$ from cliffed $W$ margin. Benzene dilution, $1060 \mathrm{minl}$. count.

\section{ANU-663.}

$$
\begin{aligned}
\delta C^{14} & =-106.6 \pm 12.2 \\
\Delta & =-111.9 \pm 12.7
\end{aligned}
$$$$
950 \pm 120
$$
lunctte. Ca. $5 \mathrm{~m}$ above lake floor. Benzene dilution, $1040 \mathrm{~min}$. count.

ANU-664.

$$
\begin{aligned}
\delta C^{14} & =-221.6 \pm 15.7 \\
\Delta & =-226.2 \pm 15.9
\end{aligned}
$$$$
2060 \pm 170
$$

Cilarcoal from Aboriginal oven on brown sand 0 lake floor. Benzene dilution, $1080 \mathrm{~min}$. count.

ANU-665.

$$
\begin{aligned}
\delta C^{14} & =-390.1 \pm 6.9 \\
\Delta & =-393.7 \pm 7.3
\end{aligned}
$$$$
4020 \pm 100
$$$$
\text { Est. } \delta C^{13}=-22 \%
$$

Charcoal from Aboriginal oven buried $50 \mathrm{~cm}$ below top of brown sand on lunette. Benzene dilution, $1040 \mathrm{~min}$. count. 
ANU-666.

$$
\begin{aligned}
\delta C^{14} & =-82.4 \pm 7.7 \\
\Delta & =-87.9 \pm 8.5
\end{aligned}
$$

$740 \pm 70$

Charcoal from Aboriginal oven on top of brown sand on lunette, overlooking lake. Benzene dilution, $1080 \mathrm{~min}$. count.
ANU-667.

$$
\begin{aligned}
\delta C^{14} & =-961.8 \pm 2.2 \\
\Delta & =-962.0 \pm 2.2
\end{aligned}
$$$$
26,270 \pm 470
$$$$
\text { Est. } \delta C^{13}=-22 \% \text { o }
$$

Charcoal from Aboriginal oven at base of greenish-gray clay sediments overlying a sandy horizon with numerous traces of Aboriginal occupation. Benzene dilution, $3120 \mathrm{~min}$. count.
ANU-668.

$$
\begin{aligned}
\delta C^{14} & =-910.3 \pm 3.9 \\
\Delta & =-910.8+39
\end{aligned}
$$
$19,420 \pm 360$

$$
\Delta=-910.8 \pm 3.9 \quad \text { Est. } \delta C^{13}=-22 \%
$$

Charcoal from Aboriginal oven dug into ancient Golgol horizon and covered by aeolian sandy clays of Zanci unit; near base of luncte overlooking lake. Benzene dilution, $3140 \mathrm{~min}$. count.
ANU-669.

$$
\delta C^{14}=-408.2 \pm 13.5
$$$$
4260 \pm 190
$$$$
\Delta=-411.7 \pm 13.6
$$$$
\text { Est. } \delta C^{13}=-22 \% \text { o }
$$

Charcoal from under baked clay mound on stabilized surface, lower part of lunette, overlooking lake. Benzene dilution, $1020 \mathrm{~min}$. count.

ANU-680.

$$
\begin{aligned}
\delta C^{14} & =-978.2 \pm 1.4 \\
\Delta & =-978.3 \pm 1.4
\end{aligned}
$$

$\mathbf{3 0 . 7 8 0} \pm \mathbf{5 2 0}$

$$
\delta C^{13}=-22.5 \pm 0.2 \%
$$

Charcoal from Aboriginal oven in gray-brown sand of Mungo unit, overlooking lake. Result is error weighted mean of 3 determinations: ANU-680/1 ( $\delta \mathrm{C}^{14}=-970.4 \pm 3.8,28,330 \pm 1100$ B.P. $) ;$ ANU $-680 / 2$ $\left(\delta \mathrm{C}^{14}=-980.3 \pm 2.1,31,590 \pm 900\right.$ B.P. $) ; \mathrm{ANU}-680 / 3\left(\delta \mathrm{C}^{14}=-978.5\right.$ $\pm 2.2,30,880 \pm 870$ B.P.); Benzene 7140 min. total count. Ages from ANU-680/1 and ANU-680/2 are from charcoal in shallow depression not covered by clay lumps. ANU-680/3 is from charcoal underneath undisturbed lumps of baked clay, used as cooking stones.

\section{ANU-681.}

\section{$\Delta=-970.5 \pm 1.5$}

Charcoal from sma hearth) in gray-brown sand of Mungo unit overlooking lake. Result is error weighted mean of 2 independent determinations: $\Lambda \mathrm{NU}-681 / \mathrm{I}$ $\left(\delta \mathrm{C}^{14}=-970.9 \pm 2.2,28,450 \pm 630\right.$ в.P. $) ; \mathrm{ANU}-681 / 2\left(\delta \mathrm{C}^{14}=-969.9 \pm\right.$ 2.1, 28,190 \pm 590 B.P.). Benzene $6160 \mathrm{~min}$. total count.

\section{ANU-682.}

$$
\begin{aligned}
\delta C^{14} & =-967.3 \pm 1.3 \\
\Delta & =-967.5 \pm 1.3
\end{aligned}
$$$$
27,530 \pm 340
$$

Charcoal from Aboriginal oven in gray-brown sand of Mungo unit, overlooking lake. Result is error weighted mean of 3 independent determinations: ANU-682/1 ( $\delta \mathrm{C}^{14}=-966.5 \pm 2.1,27,320 \pm 530$ в.r. $)$; ANU$682 / 2\left(\delta \mathrm{C}^{14}=-967.5 \pm 3.0,27,580 \pm 770\right.$ B.P. $) ;$ ANU-682/3 $\left(\delta \mathrm{C}^{14}=\right.$ $-968.1 \pm 2.2,27,720 \pm 580$ B.P.). Benzene $9180 \mathrm{~min}$. total count. Charcoal 
mixed in ash spread out in a pit. Baked clay lumps arranged on one side of pit only; archaeomagnetic studies of baked earth suggest fires at other side of pit predating oven.

ANU-683.

$$
\begin{array}{rlrl}
\delta C^{14} & =-969.2 \pm 1.5 & \mathbf{2 8 , 0 0 0} \pm \mathbf{4 1 0} \\
\Delta & =-969.4 \pm 1.5 & \text { Est. } \delta C^{1 s}=-22 \% o
\end{array}
$$

Charcoal from area of burnt earth (probably Aboriginal hearth) in gray-brown sand of Mungo unit, overlooking lake. Result is error weighted mean of 2 independent determinations: ANU-683/1 $\left(\delta \mathrm{C}^{14}=\right.$ $-967.8 \pm 2.2,27,650 \pm 560$ B.r. $) ;$ ANU $-683 / 2\left(\delta \mathrm{C}^{14}=-970.5 \pm 2.2\right.$, $28,360 \pm 620$ B.P.). Benzene $6180 \mathrm{~min}$. total count. Archaeomagnetic studies on baked earth suggest date of baking may be earlier than $\mathrm{C}^{1-1}$ age.

ANU-684.

$$
\begin{array}{rlr}
\delta C^{14} & =-798.6 \pm 13.3 & \mathbf{1 2 , 9 2 0} \pm \mathbf{5 5 0} \\
\Delta & =-799.8 \pm 13.2 & \text { Est. } \delta C^{13}=-22^{\prime \prime}
\end{array}
$$

Total organic material (including small fragments of charcoal) from small area of blackencd carth (probably Aboriginal hearth) within aeolian sediments near top of Zanci unit. Age is younger than expected. Benzene dilution, 2020 min. count.

ANU-685.

$$
\begin{array}{rrr}
\delta C^{1_{4}^{4}} & =-969.7 \pm 1.3 & \mathbf{2 8 , 1 4 0} \pm \mathbf{3 7 0} \\
\Delta & =-969.9 \pm 1.3 & \text { Est. } \delta C^{13}=-22 \% / \%
\end{array}
$$

Charcoal from under baked clay mound in gray-brown sand of lunette, overlooking lake. Result is mean of 2 independent determinations: $A N U-685 / 1\left(\delta C^{11}=-969.3 \pm 1.9,28,040 \pm 520\right.$ B.P. $)$; and ANU$6852\left(\delta C^{14}=-970.1 \pm 1.9,28,250 \pm 5.10\right.$ B.1. $)$. Benzene 6820 min. total count.

ANU-686.

$$
\begin{array}{rlr}
\delta C^{14} & =-95.9 .3 \pm 2.6 & \mathbf{2 5 , 5 7 0} \pm \mathbf{5 2 0} \\
\Delta & =-958.5 \pm 2.6 & \text { List. } \delta C^{13}=-22 \%
\end{array}
$$

Charcoal from Aboriginal oven at base of greenish-gray sandy clay overlying gray-brown sand horizon. Benzene dilution, $3100 \mathrm{~min}$. count.

$$
\begin{aligned}
& \text { ANU-687. } \\
& \begin{array}{rr}
\delta C^{14}=-987.6 \pm 2.2 \quad \mathbf{3 5 , 3 0 0} \begin{array}{r}
+\mathbf{1 5 5 0} \\
-\mathbf{1 3 0 0}
\end{array} \\
\Delta=-987.6 \pm 2.2 \quad \text { Est. } \delta C^{13}=-22 \% \text { o }
\end{array}
\end{aligned}
$$

Charcoal from blackened, friable sandy sediment of Mungo unit, possibly large Aboriginal fireplace. Benzene dilution, $3980 \mathrm{~min}$. count.

ANU 653.

\section{Lakes Leaghur and Garnpung}

$$
\text { ANU.653. } \quad \begin{array}{rlr}
\delta C^{14} & =-156.5 \pm 9.5 & \mathbf{1 4 2 0} \pm \mathbf{1 0 0} \\
\Delta & =-161.6 \pm 10.0 \quad \text { Est. } \delta C^{13}=-22 \%
\end{array}
$$

Charcoal from Aboriginal oven on floor of Lake Garnpung. Benzene dilution, $1200 \mathrm{~min}$. count. One of ca. 20 ovens. 
ANU-654.

$$
\begin{aligned}
\delta C^{14} & =-21.5 \pm 5.2 \\
\Delta & =-27 . t \pm 6.5
\end{aligned}
$$

$220 \pm 60$

Charcoal from Aboriginal oven on floor of Lake Garnpung. Benzene, $3720 \mathrm{~min}$. count. Oven $9 \mathrm{~m}$ from another dated at $1420 \pm 100$ B.P. (ANU-653).
ANU-655.

$$
\delta C^{14}=-216.1 \pm 9.7
$$$$
2010 \pm 100
$$$$
\Delta=-221.1 \pm 10.1
$$$$
\text { Est. } \delta C^{13}=-22 \%
$$

Gharcoal from Aboriginal oven on floor of Lake Garnpung. Benzene dilution, $1220 \mathrm{~min}$. count. See ANU-678 for comment.

$$
\text { ANU-674. } \quad \begin{array}{rlr}
\delta C^{14} & =-109.2 \pm 8.1 & \mathbf{9 9 0} \pm \mathbf{7 0} \\
\Delta & =-116.5 \pm 8.0 & \delta C^{13}=-20.9 \pm 0.2 \%
\end{array}
$$

Charcoal from Aboriginal oven on floor of Lake Leaghur. Benzene dilution, $1200 \mathrm{~min}$. count.

\section{ANU 675 .}

$$
\begin{aligned}
8 C^{14} & =-83.4 \pm 7.4 \\
\Delta & =-88.9 \pm 8.3
\end{aligned}
$$$$
750 \pm 70
$$$$
\text { Est. } \delta C^{13}=-22 \%
$$

Charcoal from Aboriginal oven on floor of Lake Leaghur. Benzene, $1080 \mathrm{~min}$. count.
ANU-678.

$$
\begin{aligned}
\delta C^{14} & =-257.1 \pm 6.3 \\
\Delta & =-261.6 \pm 6.9
\end{aligned}
$$$$
2440 \pm 80
$$$$
\text { Est. } \delta C^{13}=-22 \%
$$

Charcoal from Aboriginal oven set in hard clay on floor of Lake Garnpung, partly covered by sand, Benczene, 1020 min. count. From group of ovens (with ANU-65.5, $2010 \pm 100$ B.P.) exposed in a claypan scattered with microlithic stone artifacts, including pirri points. (Allen, pers. commun.).

ANU-679.

$$
\begin{aligned}
\delta C^{14} & =-330.8 \pm 7.4 \\
\Delta & =-334.8 \pm 7.8
\end{aligned}
$$$$
3270 \pm 90
$$

Charcoal from Aboriginal oven on terrace in ancient channel linking Lakes Garnpang and Leaghur. Benzene dilution, $1020 \mathrm{~min}$. count.
ANU.689.

$$
\begin{aligned}
\delta C^{4} & =-140.8 \pm 14.3 \\
\Delta & =-1+6.0 \pm 11.6
\end{aligned}
$$$$
1270 \pm 140
$$$$
\text { Est. } \delta C^{13}=-22 \% \text { o }
$$

Charcoal from Aboriginal oven on surface at W sicle of Lake Yantara

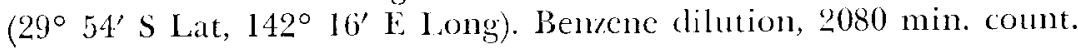

\section{Partacoona series}

Partacoona Sta. is $330 \mathrm{~km} \mathrm{~N}$ of Adelaide, South Australia on W side of Flinders Range. Samples coll. from Aboriginal ovens by M. W. McElhinny and F. E. M. Lilley, Australian Natl. Univ., and B. Powell, Partacoona Sta.

\section{ANU-656.}

$$
\begin{aligned}
\delta C^{14} & =-14.5 \pm 3.3 \\
\Delta & =-20.4 \pm 5.2
\end{aligned}
$$$$
170 \pm 60
$$

Charcoal from Aboriginal oven in gully on bank of Kanyaka Cr. $\left(32^{\circ} 05^{\prime} \mathrm{S}\right.$ Lat, $138^{\circ} 03^{\prime} \mathrm{E}$ Long). Result is error weighted mean of 2 
independent determinations from ANU-656/1 $\left(\delta \mathrm{C}^{14}=-19.1 \pm 4.2\right.$, $200 \pm 50$ в.P. $)$, and $A N U-656 / 2\left(\delta C^{1.4}=-6.1 \pm 5.6,100 \pm 60\right.$ B.P. $)$. Benzene 5980 min. total count.

ANU-657.

$$
\begin{aligned}
\delta C^{14} & =-105.6 \pm 38.3 \\
\Delta & =-111.0 \pm 38.1
\end{aligned}
$$

$940 \pm 350$

Est. $\delta C^{13}=-22 \%$

Charcoal from Aboriginal oven covered by soil in gully on $\mathrm{E}$ bank of Kanyaka Cr. ( $32^{\circ} 03^{\prime} \mathrm{S}$ Lat, $138^{\circ} 05^{\prime} \mathrm{E}$ Long). Benzene dilution, 2260 min. count.

\section{ANU-658.}

$$
\begin{aligned}
\delta C^{14} & =-100.7 \pm 9.3 \\
\Delta & =-106.1 \pm 10.0
\end{aligned}
$$

$$
\begin{array}{r}
900 \pm 90 \\
\text { Est. } \delta C^{13}=-22^{\%} \%
\end{array}
$$

Charcoal from Aboriginal oven exposed in cliffed $\mathrm{E}$ bank of Kanyaka Cir. Ca. $7 \mathrm{~m}$ above present river level and $50 \mathrm{~cm}$ below top of bank. Ca. $30 \mathrm{~m} \mathrm{~N}$ from ANU-657. Benzene dilution, $1060 \mathrm{~min}$. count.

ANU-673.

$$
\begin{aligned}
\delta C^{14} & =+34.7 \pm 40.0 \\
\Delta & =+28.5 \pm 40.0
\end{aligned}
$$

$>$ Modern

Clarconl from Aboriginal oven overlooking

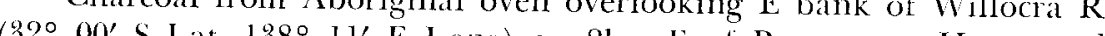
(32. $50^{\prime} S$ Lat, $138^{\circ} 11^{\prime}$ E Long) cal. $2 \mathrm{~km} \mathrm{E}$ of Partatcoona Homestcad. Benzene clilution, $2260 \mathrm{~min}$. count.

\section{Murray River series}

Several burnt tree stumps surrounded by baked earth were shown to one of the authors (M.B.) by J. Urquhart, Australian Natl. Univ. The sites are in fluviatile sediments, and have been useful in studies of the history of the Murray R. (J. Urquhart, ms. in preparation), as well as archaeomagnetic investigations. The Aboriginal ovens (ANU-676, $.67 i$ ) are ca. $20 \mathrm{~km}$ from present-day Murray R.

\section{ANU-676.}

$$
\begin{aligned}
\delta C^{14} & =-207.5 \pm 17.7 \\
\Delta & =-209.1 \pm 18.0
\end{aligned}
$$

$1880 \pm 180$

Charcoul from Aboriginal oven on I reto Sta min. count. Coll. by D. Lelwards.

$$
\text { ANU-677. } \quad \begin{array}{rlrl}
\delta C^{14} & =-256.5 \pm 12.6 & \mathbf{2 4 0 0} \pm \mathbf{1 4 0} \\
\Delta & =-258.0 \pm 12.9 & \text { Est. } \delta C^{13} & =-24^{\prime} / \mathrm{co}
\end{array}
$$

Charcoal from Aboriginal oven $(0.5 \mathrm{~km}$ NW of ANU-676) Benzene dilution, $1120 \mathrm{~min}$. count. Sample contained some very fine rootlets. Coll. by D. Edwards.

ANU-690.

$$
\begin{aligned}
\delta C^{14} & =-158.8 \pm 11.4 \\
\Delta & =-160.4 \pm 11.8
\end{aligned}
$$

$$
1400 \pm 110
$$

Charcoal from under lumps of baked clay $5 \mathrm{~m}$ below top Victoria bank of Murray R. ( $34^{\circ} 44^{\prime}$ S Lat, $143^{\circ} 07^{\prime}$ E Long). Benzene dilution, 2000 min. count. 
ANU-691.

$$
\begin{aligned}
8 C^{14} & =-46.1 \pm 6.7 \\
\Delta & =-48.0 \pm 7.7
\end{aligned}
$$

$400 \pm 70$

Charcoal from burnt tree stump $2 \mathrm{~m}$ below top of New South Wales bank of Murray R. (95 $55^{\circ} \mathrm{S}$ Lat, $145^{\circ} 25^{\prime} \mathrm{E}$ Long). Benzene, 1000 min. count.

$$
\text { ANU-692. } \quad \begin{array}{rlrl}
\delta C^{14} & =-533.7 \pm 8.9 & \mathbf{6 1 4 0} \pm \mathbf{1 6 0} \\
\Delta & =-531.6 \pm 9.1 & \text { Est. } \delta C^{13}=-21 \%
\end{array}
$$

Charcoal from burnt tree stump $6 \mathrm{~m}$ below top of Victoria bank of Murray R. (35 $56^{\prime} \mathrm{S}$ Lat, $144^{\circ} 28^{\prime} \mathrm{E}$ I.ong). Benzene dilution, 2140 min. count

ANU-693.

$$
\begin{array}{rrr}
\delta C^{14} & =-481.5 \pm 5.4 & \mathbf{5 2 9 0} \pm \mathbf{9 0} \\
\Delta & =-782.5 \pm 5.8 & \text { Est. } \delta C^{13}=-2.4 \%
\end{array}
$$

Charcoal from area of burnt earth, $2 \mathrm{~m}$ below top of Victoria bank of Murray R., 70m downstream from ANU-692. Benzene, $1000 \mathrm{~min}$. count.

\section{Keilor series}

Soil pit operators have uncovered areas of burnt earth in terrace silts (Holocene age) of Marybyrnong R. near Keilor, Victoria. These sites appear to have been disturbed by ancient floods which deposited silt on them (ANU-652, -694, -695).

A section of Pleistocone Maribyrnong $R$. sediments is exposed in Dry Creek, near its confluence with Maribyrnong. Several excavations are being made by $A$. Gallus and the Archaeol. Soc. Victoria in ancient river sediments and a series of channel cleposits cut into those sediments (Gallus, 1971). Excavation A (Gallus, unpub.) is in one of these channels. Yellow-gray (KW) clay is unconformably overlain by chocolate (A) clay.

\section{ANU-652.}

$$
\begin{array}{rlr}
\delta C^{14} & =-570.9 \pm 23.9 & \mathbf{6 8 1 0} \pm \mathbf{4 6 0} \\
\Delta & =-571.7 \pm 23.9 & \text { Est. } \delta C^{13}=-2+\%
\end{array}
$$

Charcoal mixed with burnt earth uncovered by front-end loader in soil pit at Green Gully, Keilor ( $37^{\circ} 44^{\prime} \mathrm{S}$ Lat, $144^{\circ} 49^{\prime} \mathrm{E}$ Long). Benzene dilution, $1100 \mathrm{~min}$. count. Coll. by D. J. Mulvaney and M. W. McLlhinny.

ANU-694.

$$
\begin{array}{rrr}
\delta C^{14} & =-498.8 \pm 5.3 & \mathbf{5 5 7 0} \pm \mathbf{9 0} \\
\Delta & =-499.8 \pm 5.7 & \text { Lst. } \delta C^{13}=-24 / c 0
\end{array}
$$

Charcoal mixed with burnt earth $2 \mathrm{~m}$ below surface in terrace sediments, $4 \mathrm{~lm}$ from E bank of Marybyrnong R. $\left(37^{\circ} 42^{\prime} \mathrm{S} \mathrm{Lalt,} 144^{\circ} 50^{\prime}\right.$ E Long). Benzene, $1020 \mathrm{~min}$. count.
ANU-695.

$$
\delta C^{14}=-562.9 \pm 5.4
$$$$
6660 \pm 110
$$$$
\Delta=-563.8 \pm 5.7
$$$$
\text { Est. } \delta C^{13}=-2.1 \%
$$

Charcoal mixed with burnt earth $5 \mathrm{~m}$ below surface, exposed in soil pit. Ca. $11 \mathrm{~m}$ from ANU-694, towards Marybynnong R. Benzene dilution, $2040 \mathrm{~min}$. count. 
ANU-696.

$$
\begin{aligned}
\delta C^{14} & =-992.2 \pm 3.2 \\
\Delta & =-992.2 \pm 3.2
\end{aligned}
$$

$>\mathbf{3 1 , 1 8 0}$

Est. $\delta C^{13}=-24 \%$ o

Large piece of charcoal embedded $5 \mathrm{~cm}$ below top of yellow-gray (KW) clay in Excavation A, Dry Creek. Sample treated with hot $2 \%$ $\mathrm{NaOH}$ solution to remove any possible humic acid contamination. Benzene dilution, $4000 \mathrm{~min}$. count. Measured activity is within $\mathrm{C}^{14}$ dating limits at $95 \%$ confidence level. Indicated age $39,000+2260$ B.P.

\section{ANU-697.}

$$
\begin{array}{rlr}
\delta C^{14} & =-960.8 \pm 6.3 & \mathbf{2 6 , 0 3 0} \\
\Delta & =-960.8 \pm 6.3 \quad \text { Est. } \delta C^{13}=
\end{array}
$$

$\mathrm{NaOH}$ soluble fraction of charcoal from presumed human hearth (Gallus, pers. commun.) in chocolate clay of Excavation A. Charcoal fragments showed wood-grain texture, with humic acid stains spreading into clay around each pellet. Sample exhaustively leached in hot $2 \% \mathrm{NaOH}$, large soluble fraction recovered, acidified, and precipitate coll. for $\mathrm{C}^{1.1}$ analysis. Benzene dilution, $3180 \mathrm{~min}$. count. Charcoal appears to have clecayed in situ, with clay sediment around fireplace preventing appreciable movement of humic acid; date therefore considered reliable. Coll. by A. Gallus.

\section{RFFERENCES}

Barbetti, M. and Allen, II., 1972, Prehistoric man at Lake Mungo, Australia, by 32.000 yr. B.P.: Nature, v. 240 , p. $46-48$

Barbetti, M. and McElhinny, M., 1972, Evidence for a geomagnetic excursion 30,000 yr 13.1.: Nature, v. 299 , p. $327-330$

Bowler, J. M., 1971, Pleistocene salinities and climatic change: evidence from lakes and lunctes in southeastern Australia: in, D. J. Mulvancy and $\mathrm{J}$. Golson (edts.) Aboriginal man and environment in Australia, Camberra, Australian Natl. Univ. Press, p. 47-65.

Gallus, A., 1971, Excavations at Keilon: The Artefact, newsletter no. 24.

Polach, H. A., 1969, Optimisation of liquid scintillation radiocarbon age determinat tions and reporting of ages: Atomic linergy in Australia, v. 12, no 3, p. 21-28.

1972, Crosschecking of NBS oxalic acid and secondary laboratory radiocarbon dating standards: Intematl. ratliocarbon dating conf. Proc., New Zealand, October 1972, p. H92-H120.

Polach, H. A., Gower, John, and Fraser, Ian, 1972: Synthesis of high purity benzene for radiocarbon dating by the liquid scintillation method: Internatl. radiocarbon dating conf. Proc., New /caland, October 1972, p. B35-1349.

Polach, H. A., Iovering, J. F., and Bowler, J. M., 1970, $\mathrm{NNU}$ radiocarbon date list IV: Radiocarbon, v. 12, p. 1-18. 